\title{
IMPLEMENTATION OF ANFIS FOR GPS-AIDED INS UAV MOTION SENSING AT SHORT TERM GPS OUTAGE
}

\author{
Chot Hun Lim, Tien Sze Lim and Voon Chet Koo \\ Faculty of Engineering and Technology, Multimedia University, Melaka, Malaysia
}

Received 2014-08-05; Revised 2014-08-26; Accepted 2014-10-14

\begin{abstract}
The recent improvement in Micro-Electro-Mechanical System (MEMS) technology has enabled the evolvement of Inertial Navigation Unit (INU) to be built on top of a low cost, small size Integrated Circuit (IC) chip. Due to the nature of the MEMS INU, its outputs are normally corrupted by the resided stochastic noise. A common practice to regulate its measurements into usable motion data is by fusing the Global Positioning System (GPS) measurement data with the MEMS INU measurement data through Kalman filter for position, velocity and orientation estimations. Such integrated system is known as GPS-aided Inertial Navigation System (INS). Note that the robustness of the GPS-aided INS relies heavily on the availability of the GPS signals. In the event of no GPS signals, the overall system will solely depend on the INU to predict the position, velocity and orientation. The prediction results will eventually drift from its true value due to the INU's resided stochastic noise. In this study, a remedy system using Adaptive Neuro-Fuzzy Inference System (ANFIS) is developed to improve the performance of the GPS-aided INS during GPS outage condition. UAV motion sensing experiment was carried out and GPS outage conditions were imposed at several locations during the UAV navigation. The motion prediction dataduring GPS outages, with and without ANFIS implementation, were compared and the results clearly show that the GPS-aided INS with ANFIS implementation achieved better performance than the GPS-aided INS without ANFIS.
\end{abstract}

Keywords: ANFIS, GPS, INS, UAV

\section{INTRODUCTION}

Inertial Navigation System (INS) is a type of deadreckoning system that utilized Inertial Navigation Unit (INU) for three dimensional position, velocity and orientation estimations (Titterton and Weston, 1997). With recent advancement in Micro-ElectroMechanical System (MEMS), the INU is able to be built on top of a small size, low cost Integrated Circuit (IC) chip (Nebot and Durrant, 1999). However, such INU configuration still fail to work as a standalone device for navigation applications due to its resided stochastic noise (El-Diasty and Spiros, 2008; Sheimy et al., 2008; Lim et al., 2012a). A conventional approach to solve this issue is to fuse the Global Positioning System (GPS) data with the INU measurement using Kalman filter, where such configuration is commonly known as GPS-aided INS (Alison, 2005; David et al., 2006).

One of the major issues of GPS-aided INS is that such system, when operates without GPS data, solely depends on INU measurements in which its predictions will eventually diverged from the true values (Sameh, 2003). Temporal no GPS condition may occur due to signal blockage or intentional signal jamming (Adrian et al., 2004). Under such condition the accuracy of position estimation will be degraded. Recent study by Lim et al. (2014) suggested that the Unmanned Aerial Vehicle (UAV) motion sensing, under no GPS condition, suffered significant drifts. Two different stochastic noise models, namely the Gauss-Markov (GM) model and the Autoregressive (AR) model, were implemented as the GPS-aided INS noise model. Although results indicated Corresponding Author: Chot Hun Lim, Faculty of Engineering and Technology, Multimedia University, Melaka, Malaysia 
that the GPS-aided INS with AR model operated better than system with GM model during no GPS condition, the accumulated errors for the system with AR model were still significant.

This study presents a design that reduced the above mentioned accumulation errors by integrating the GPS-aided INS with an Adaptive Neuro-Fuzzy Inference System (ANFIS) for UAV motion sensing. ANFIS was proposed by Jang (1993) as a new adaptive fuzzy system with self-learning and selftuning features. As reported by Jang (1993), ANFIS generates similar results as compared to conventional Neural Networks, with advantages such as less processing time and real-time realization over the Neural Network. In terms of operation, the ANFIS is trained during the present of GPS data using the GPSaided INS inputs and outputs information. During the absent of GPS data, the ANFIS is temporary replacing the GPS to aid the INU for motion sensing.

The remaining of this study is outlined as follows. Section 2 describes the GPS-aided INS operational structure, with elaborations on the navigation equations (Titterton and Weston, 1997) and the Kalman filtering process. Section 3 discusses the ANFIS fundamentals and its implementation for temporal integration of GPS-aided INS. Section 4 presents the UAV experiment and the produced results. Intentional no GPS conditions are implemented on several locations in the UAV's flight path and the generated results are compared among the GPS-aided INS with and without ANFIS. Lastly, Section 5 concludes the findings.

\section{GPS-AIDED INS}

A typical GPS-aided INS consists of two important elements; the GPS that provides dead reckoning position measurements and the INU with three dimensional accelerometers, gyroscopes and magnetometers for position, velocity and orientation estimations (Lim et al., 2012b). Kalman filter is implemented to fuse the data from these two elements for motion sensing. Note that Kalman filtering process has two important phases, namely the prediction phase that utilized the INU data and the measurement phase that utilized the GPS data.

Figure 1 shows the operational block diagram of the GPS-aided INS. The MEMS inertial sensor consists of three dimensional accelerometers, gyroscopes and magnetometers. A pre-processing stage which involved wavelet denoising (Naser et al., 2004) is applied on the accelerometers and gyroscopes raw data. The pre-processed accelerometers and gyroscopes data is fed into the navigation equation to calculate the predicted position $\hat{r}_{k}^{n}$, velocity $\hat{v}_{k}^{n}$ and orientation $\hat{C}_{b, k}^{n}$, as shown in Equation 1:

$$
=\left(\begin{array}{c}
\hat{r}_{k-1}^{n}+\hat{v}_{k-1}^{n} \cdot \Delta t \\
\hat{v}_{k-1}^{n}+\left[\hat{C}_{b, k-1}^{n} s_{k-1}^{b}-\omega_{i n}^{n} \times \hat{v}_{k-1}^{n}+g^{n}\right] \cdot \Delta t \\
\hat{C}_{b, k-1}^{n} \cdot \exp \left\{\Omega_{i b}^{b}-\Omega_{i n}^{b}\right\} \cdot \Delta t
\end{array}\right)
$$

Where:

$\omega_{i n}^{n}=$ The Earth's coriolis rate $(\mathrm{rad} / \mathrm{s})$

$\omega_{i b}^{b}=$ The gyros measurement in body frame $(\mathrm{rad} / \mathrm{s})$

$\omega_{i n}^{b}=$ The Earth's rotational rate $(\mathrm{rad} / \mathrm{s})$

$\Omega_{i b}^{b}=$ The skew symmetry matrix of $\omega_{i b}^{b}(\mathrm{rad} / \mathrm{s})$

$\Omega_{i n}^{b}=$ The skew symmetry matrix of $\omega_{i n}^{b}(\mathrm{rad} / \mathrm{s})$

$\Delta \mathrm{t}=$ The discrete sampling time (s)

Equation1 measurements are corrupted by deterministic errors and stochastic errors (Lim et al., 2012a). Note that the deterministic errors can be removed through proper calibrations. Stochastic errors, on the other hand, are random errors that can be predicted through static experiments (Nebot and Durrant, 1999; Sameh, 2003). The influence of the stochastic errors in Equation 1 can be observed by performing dynamic perturbation on Equation 1 and its dynamic perturbed error Equation is shown in Equation 2 to 4 :

$$
\begin{aligned}
& \delta x_{k}\left(\begin{array}{l}
\delta \hat{r}_{k}^{n} \\
\delta \hat{v}_{k}^{n} \\
\varepsilon_{b, k}^{n}
\end{array}\right)=\phi_{k-1} \cdot \delta x_{k}+W_{k-1} \\
& \psi_{k-1} \approx I_{9 \times 9}+A_{k-1} \\
& A_{k-1}=\left(\begin{array}{llc}
A_{r r, k-1} & A_{r v, k-1} & 0_{3 \times 3} \\
A_{v r, k-1} & A_{v v, k-1} & \left(s_{k-1}^{n} \times\right) \\
A_{e r, k-1} & A_{e v, k-1} & -\left(\omega_{i n, k-1}^{n} \times\right)
\end{array}\right)
\end{aligned}
$$

\section{Where:}

w.r.t. = Refer to "with respect to"

$\phi_{k-1}=$ The transition equation expressed in Equation 3

$W_{k-1}=$ The stochastic errors of accelerometers $\left(\mathrm{m} / \mathrm{s}^{2}\right)$ and gyroscopes ( $\mathrm{rad} / \mathrm{s})$

$A_{r r, k-1}=$ The Jacobian of position w.r.t. position

$A_{r r, k-1}=$ The Jacobian of position w.r.t. velocity

$A_{v r, k-1}=$ The Jacobian of velocity w.r.t. position 
$A_{v v, k-1}=$ The Jacobian of velocity w.r.t. velocity

$A_{e r, k-1}=$ The Jacobian of orientation w.r.t. position

$A_{e v, k-1}=$ The Jacobian of orientation w.r.t. velocity

$\mathrm{I}_{9 \times 9}=$ A $9 \times 9$ identity matrix

$0_{3 \times 3}=\mathrm{A} 3 \times 3$ zero matrix

$\left({ }^{*} \times\right)=$ The cross product

Equation 2 denotes the dynamic error equations of Equation 1 in state space model. Such model is to be substituted into the Kalman filtering process for the prediction phase. On the other hand, the Kalman filter measurement phase consists of the measurement equations as shown in Equation 5 and 6:

$z_{k}=H_{k} \delta \hat{x}_{k}+\square_{k}$

$\delta \tilde{x}_{k}=\left(\begin{array}{l}\tilde{r}_{k}^{n}-\tilde{r}_{k, G P S}^{n} \\ \hat{v}_{k}^{n}-\hat{v}_{k, G P S}^{n} \\ \hat{\vartheta}_{k}^{n}-\hat{\vartheta}_{k, M A G}^{n}\end{array}\right)$

Where:

$z_{k} \quad=$ The measurement update

$H_{k} \quad=$ The measurement Jacobian

$Q_{k} \quad=$ The random measurement noise response

$\delta \tilde{x}_{k} \quad=$ The observation errors

$\tilde{r}_{k, G P S}^{n}=$ The position measurements from GPS (m)

$\hat{v}_{k, G P S}^{n}=$ The velocity measurements from GPS $(\mathrm{m} / \mathrm{s})$

$\hat{\vartheta}_{k, M A G}^{n}=$ The orientation measurements derived from magnetometers (rad)

The Kalman filter fuses the dynamic error equations (Equation 2) with the measurement equations (Equation 5) to calculate the best errors estimation in term of three dimensional position $\delta \tilde{r}_{k}^{n}$, velocity $\delta \tilde{v}_{k}^{n}$ and orientation $\tilde{\varepsilon}_{b, k}^{n}$. As referred to Fig. 1, these calculated errors are used to compensate the computation of motion data through the subsequent INU measurements in the navigation equations.

\section{ANFIS AND ITS IMPLEMENTATION IN GPS-AIDED INS}

This section outlines the introduction of ANFIS and the detail implementation of ANFIS to the GPS-aided INS during GPS outage conditions.

\subsection{Fundamental of ANFIS}

The Adaptive Neuro-Fuzzy Inference System, or ANFIS, was originally proposed by Jang (1993) as a new adaptive fuzzy system. It is a special fuzzy inference system that possesses self-learning and self-tuning abilities given with sufficient input-output data. Fig. 2 shows a typical ANFIS network architecture of two inputs and one output with three fuzzy rules. Generally the overall ANFIS network can be separated into $L$ distinct layers, with each layer holds a certain number of nodes with specific operation. Hence the $k$-th layer's $i$-th node can be expressed as $(k, i)$ and its corresponding node function as $O_{i}^{k}$. Detailed descriptions of each layer can be found in Jang (1993) work.

\subsection{ANFIS Algorithm}

The ANFIS process involves fine-tuning and improving an initial fuzzy inference system model through iterative self-adaptive learning algorithms, where the initial fuzzy inference system model is normally defined by the ANFIS as an approximate fuzzy model. The self-adaptive training process consists of multiple iterations or epochs to improve and optimize the consequent and premise parameters (Jang, 1993). The purpose of training iteration is to reduce the measured error, where the error is defined as the sum of squared difference between the actual output and the desired output. The training iteration will be eventually halted with two conditions, either the number of iteration reaches a predefined number or the obtained error is lower or equal to the predefined error.

ANFIS utilizes a hybrid learning rule that consists of gradient descent back propagation technique and the least mean square algorithm for the tuning of ANFIS premise parameters and consequent parameters to achieve optimal output predictions. Fig. 3 depicts the ANFIS block diagram incorporated with the gradient descent algorithm and the least mean square algorithm.

\subsection{ANFIS Implementation}

The GPS-aided INS shown in Fig. 1 provides accurate measurements only with the presence of GPS data. Despite the availability of GPS signals under most situations, there exist several extreme conditions where the GPS signals might be temporarily unavailable, such as signal blockage due to obstacle, intentional signal jamming or interference caused by unknown source. In terms of equation representation, the measurement Jacobian $\mathrm{H}_{\mathrm{k}}$ in Equation 5 can be elaborated as: 


$$
H_{k}=\left(\begin{array}{ccc}
D . G P S_{\text {exist }} & 0_{3 \times 3} & 0_{3 \times 3} \\
0_{3 \times 3} & I_{3 \times 3} \cdot G P S_{\text {exist }} & 0_{3 \times 3} \\
0_{3 \times 3} & 0_{3 \times 3} & I_{3 \times 3}
\end{array}\right)
$$

Where:

$$
\begin{aligned}
G P S_{\text {exist }}= & \text { The flag signifies whether the GPS signal is } \\
& \text { available or absent } \\
D= & \text { The transformation matrix from Earth's } \\
& \text { coordinate (Longitude, Latitude, Altitude) } \\
& \text { into Navigation coordinate }(\mathrm{m})
\end{aligned}
$$

As refer to Equation 5 and 7, under no GPS condition, the measurement update $z_{k}$ will have no updates on the overall Kalman filter's measurement phase. This will force the Kalman filter to solely depend on the prediction phase to estimate the predicted errors. The estimated outputs without GPS data will eventually diverged from the true value. Hence ANFIS is proposed to temporary replaced the GPS during no GPS data condition. Rather than taking over the GPS, the ANFIS merely act as a remedy system to aid the measurement phase of Kalman filter at GPS outage situation.

ANFIS needs to be trained before any utilization can be taken place. The operation of ANFIS is separated into three distinct functions, namely the startup operation, the training operation and the prediction operation. The startup operation involves setting up an initial ANFIS fuzzy set model, while the training operation trains the ANFIS fuzzy set's premise parameters and consequent parameters through the input-output pairs of data and the prediction operation is utilized only at GPS outage condition. Fig. 4 shows block diagram of ANFIS implementation in the GPS-aided INS. In the beginning the ANFIS model is initialized through an initial model, normally a Takagi-Sugeno model. Next the ANFIS model is trained by utilizing the input-output data pairs. Here the training input is selected to be the estimated position derived during the present of GPS signal and the training output is the estimated position error. The training will continued until reaching a predefined number of epochs or achieving certain minimum RSME. Notice that the training process will continue as long as the GPS signal is present. Lastly, the training operation will halt during GPS outage condition and the prediction operation will be handled by the latest updated ANFIS model.

\subsection{ANFIS Setup and Considerations}

The computational power of ANFIS training process should be taking into account for real-time system implementation. Several key parameters have to be considered for efficient ANFIS training:
- The sliding window size

- Number of epochs (or training iteration)

- The Root-Mean-Square-Error (or RMSE)

- The training step size

In common practice, a sliding window together with the cross-validation technique is adopted to ensure realtime system's robustness (Amari et al., 1997). The crossvalidation technique is a process of partitioning a relatively large sample of data into small groups of subsample such that the analysis is focused in one subsample while further subsamples are assumed to be temporarily absent. As such, the size of the sliding window emerged as a crucial parameter in data partitioning. A large window size offers sufficient sample size for ANFIS training which improves the module performance, but in return it consumes a large computational power which could potentially influence the real-time processing. On the other hand, a small window size requires less processing power in ANFIS training, thus ensuring smooth operation of real-time processing. However, small window size may degrade system performance during prediction operation.

The number of epochs refers to the ANFIS training repetition towards a set of input-output pairs. Each training epoch generates a RMSE, computed from the difference between the trained outputs with the actual outputs data. The smaller the RMSE the better the trained ANFIS is. Theoretically more training epochs will result in producing better RMSE. In practice, however, the training iteration is a finite number and its value should be a logical number after taking into consideration on the computational time requirement. In general, both the RMSE and the number of epochs should be determined accordingly, with the number of epochs is set to 100 and the RMSE is set to $10^{-4}$ (Rashad et al., 2007).

The ANFIS step size emerged as an important factor that influence the performance of the ANFIS training. The parameters that influence the ANFIS training process are the initial step size, the increase rate and decrease rate of step size. The step size is commonly used to represent the length of each gradient transition, with its initial value being modified by either the increase rate or the decrease rate during the ANFIS training. Fig. 5 outlines the RMSE plots with respect to 20 s window size (Rashad et al., 2007) for x-position component for eight different initial step sizes of 0.001 , $0.005,0.01,0.5,1,5,10$ and 25 , respectively. Note that $20 \mathrm{~s}$ of training window size is chosen since this window size possess adequate training samples for ANFIS while in the same time small enough to ensure smooth real-time processing (Ahmed et al., 2009). 


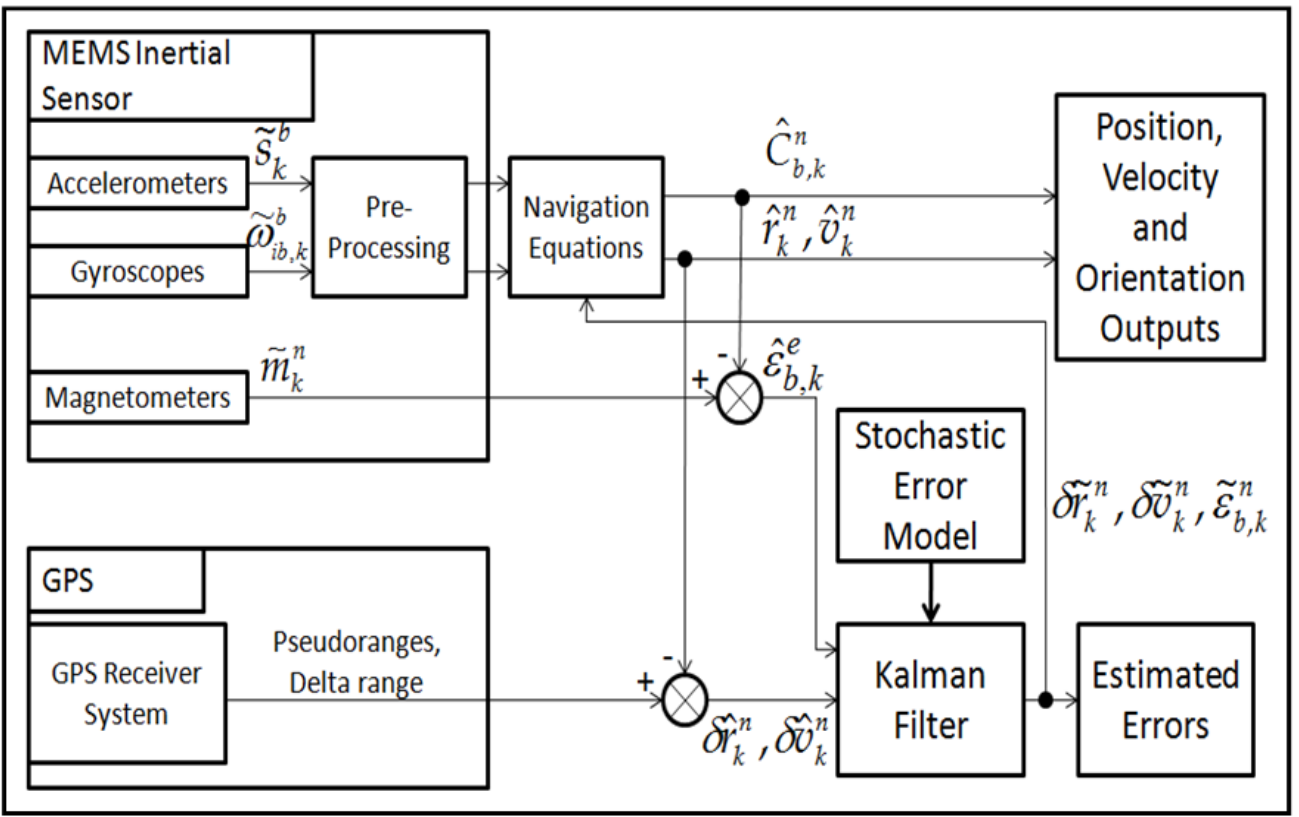

Fig. 1. GPS-Aided INS block diagram

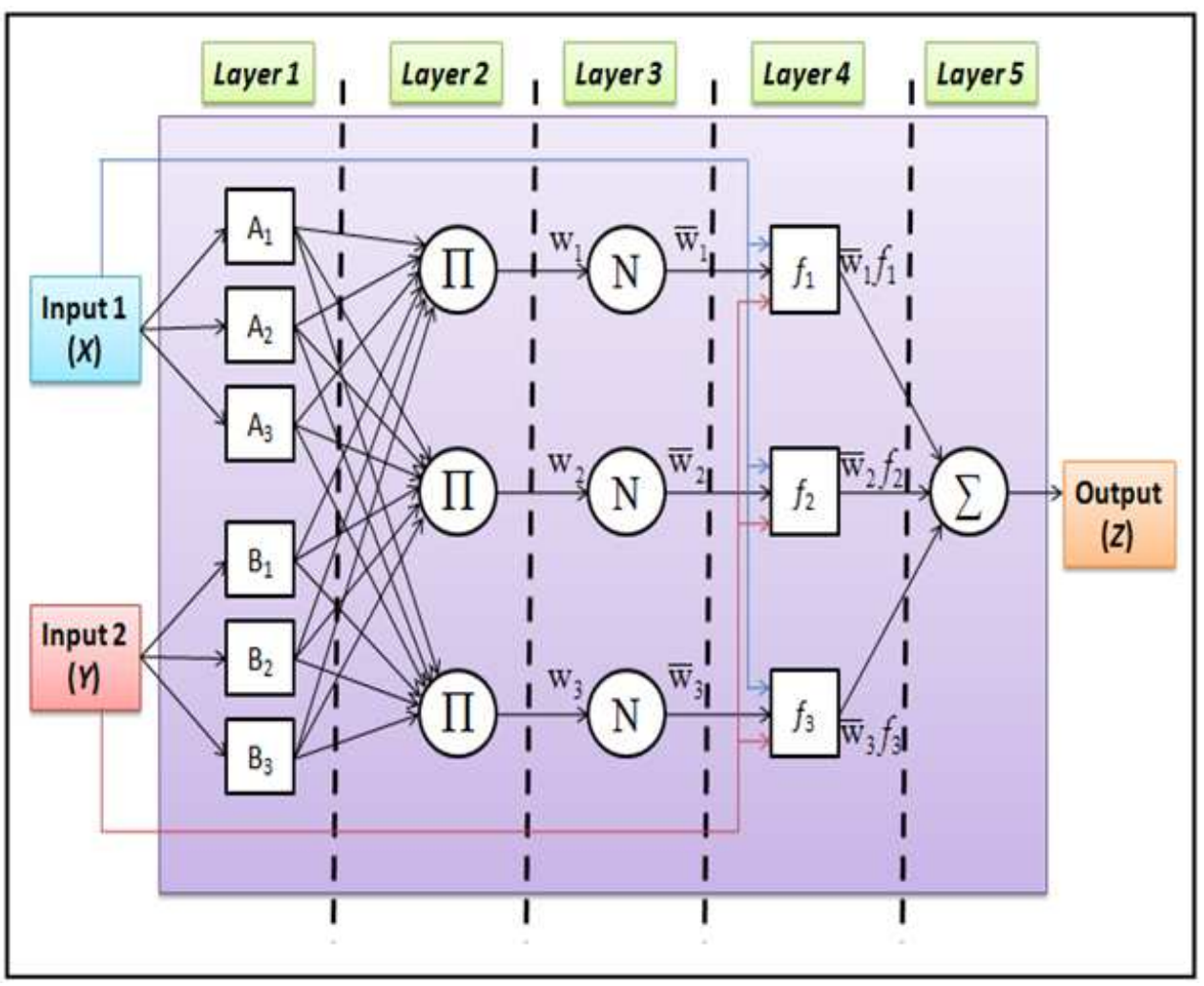

Fig. 2. A typical ANFIS network architecture 
Chot Hun Lim et al. / Journal of Computer Science 10 (12): 2564.2575, 2014

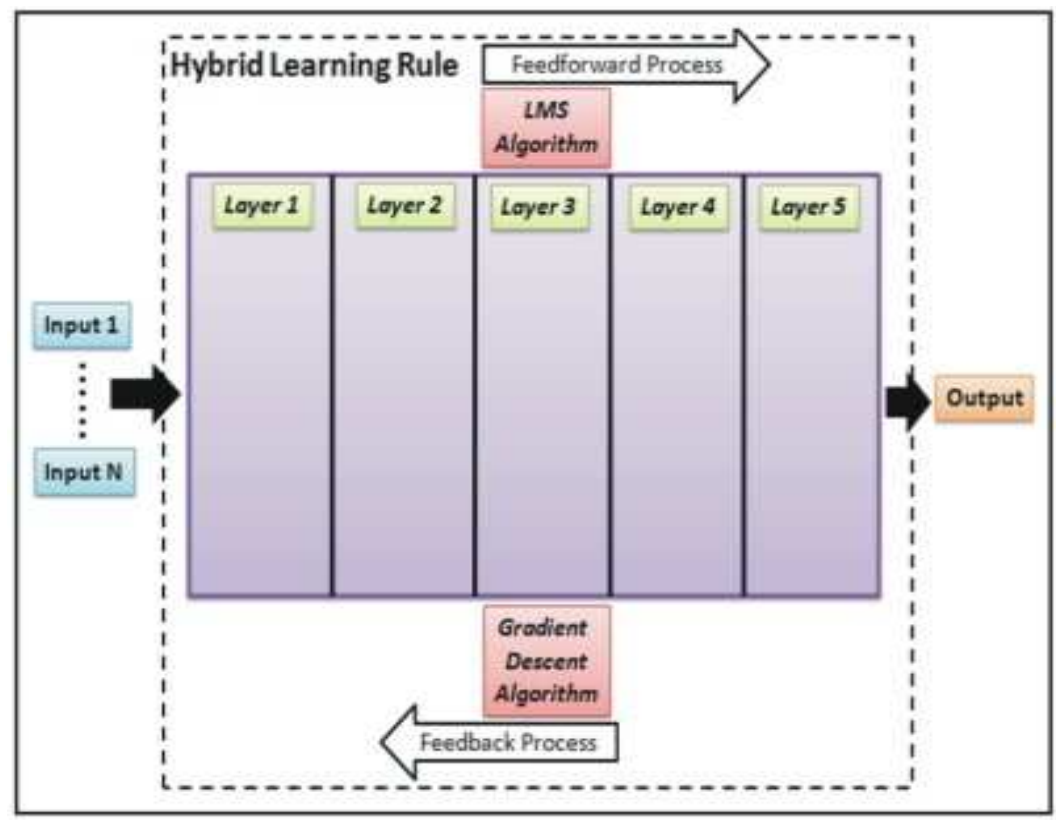

Fig. 3. Block diagram of ANFIS with feedback learning algorithms

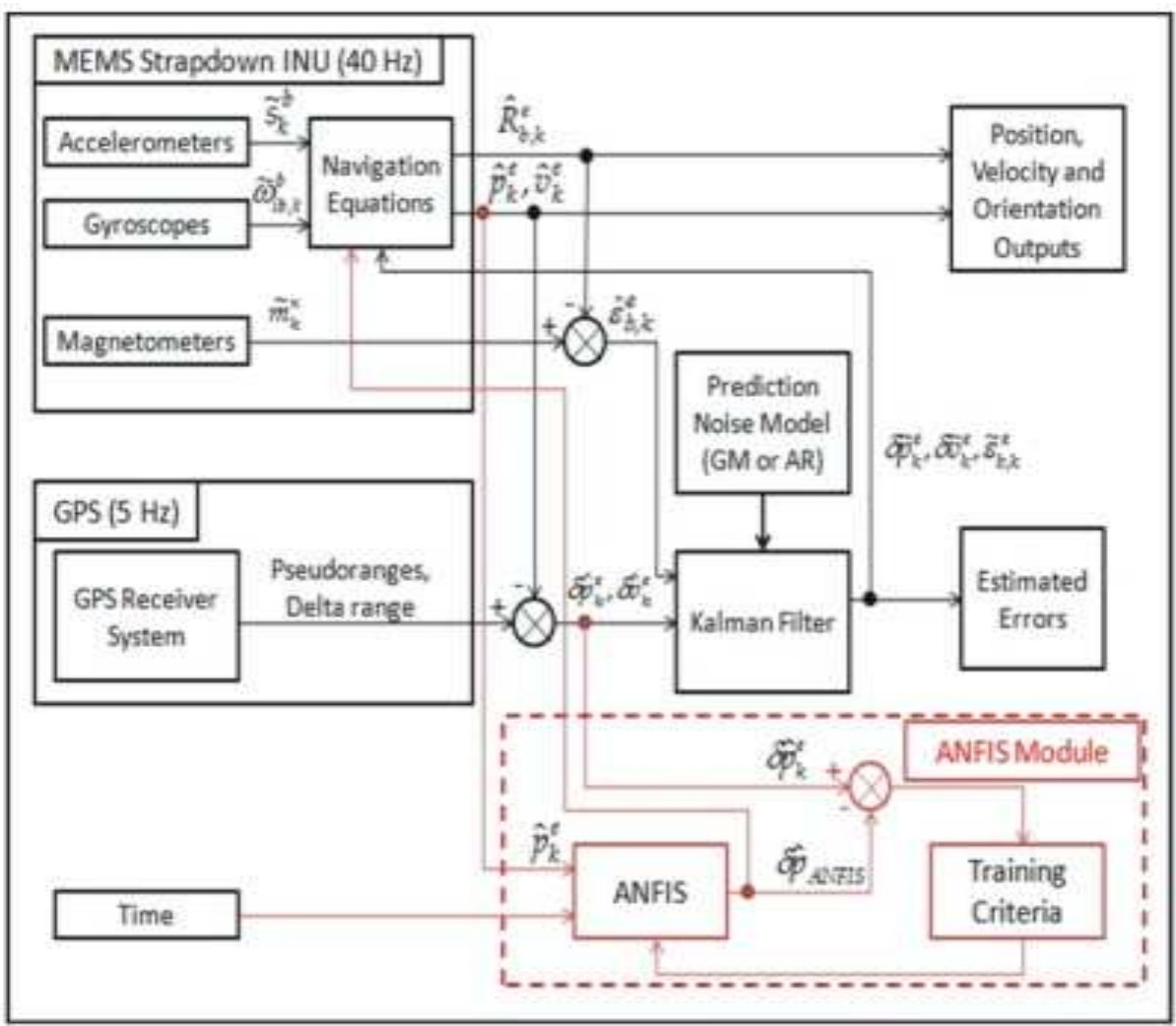

Fig. 4. Block diagram of ANFIS implementation in the GPS-aided INS 
Chot Hun Lim et al. / Journal of Computer Science 10 (12): 2564.2575, 2014
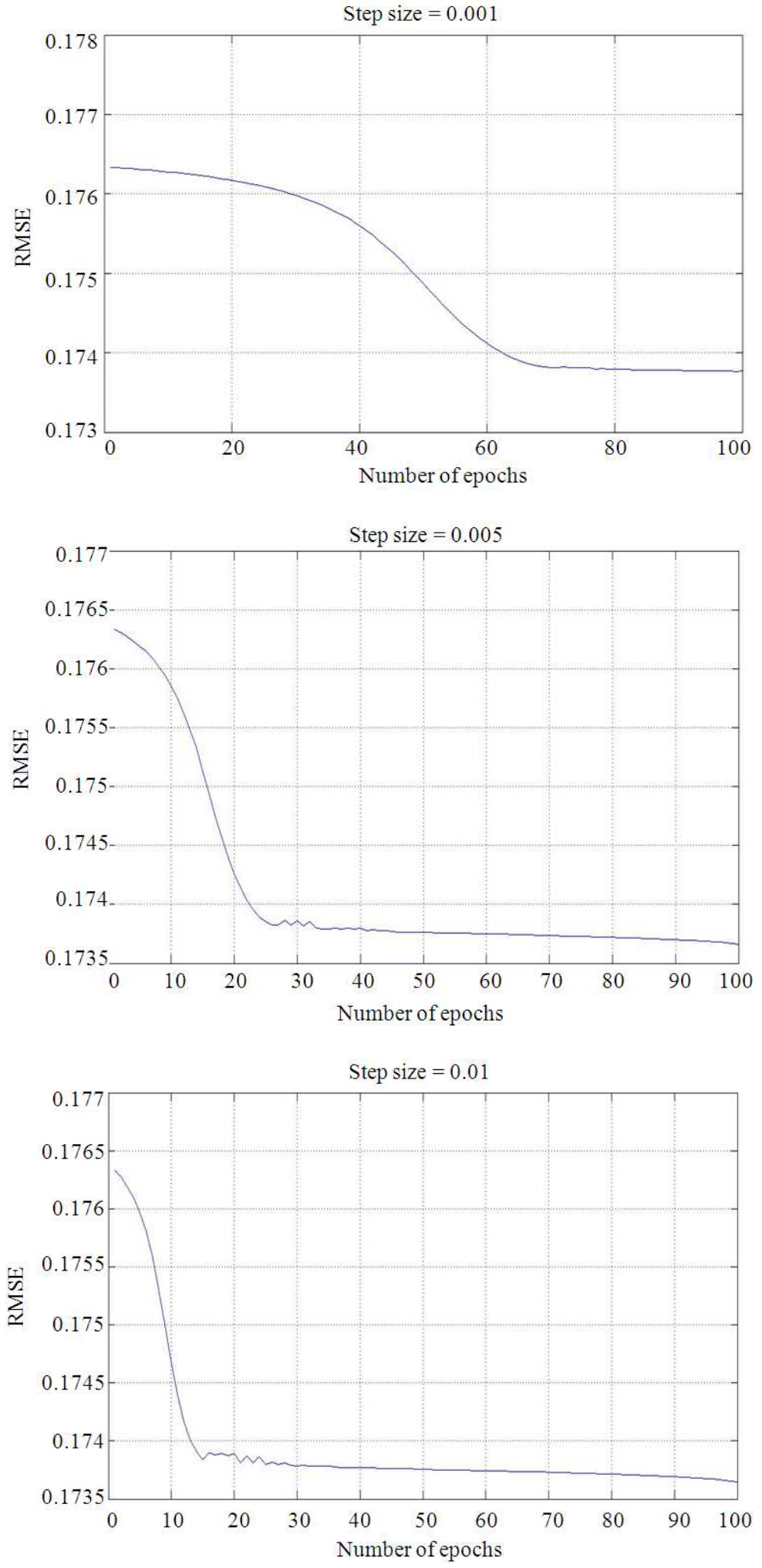
Chot Hun Lim et al. / Journal of Computer Science 10 (12): 2564.2575, 2014
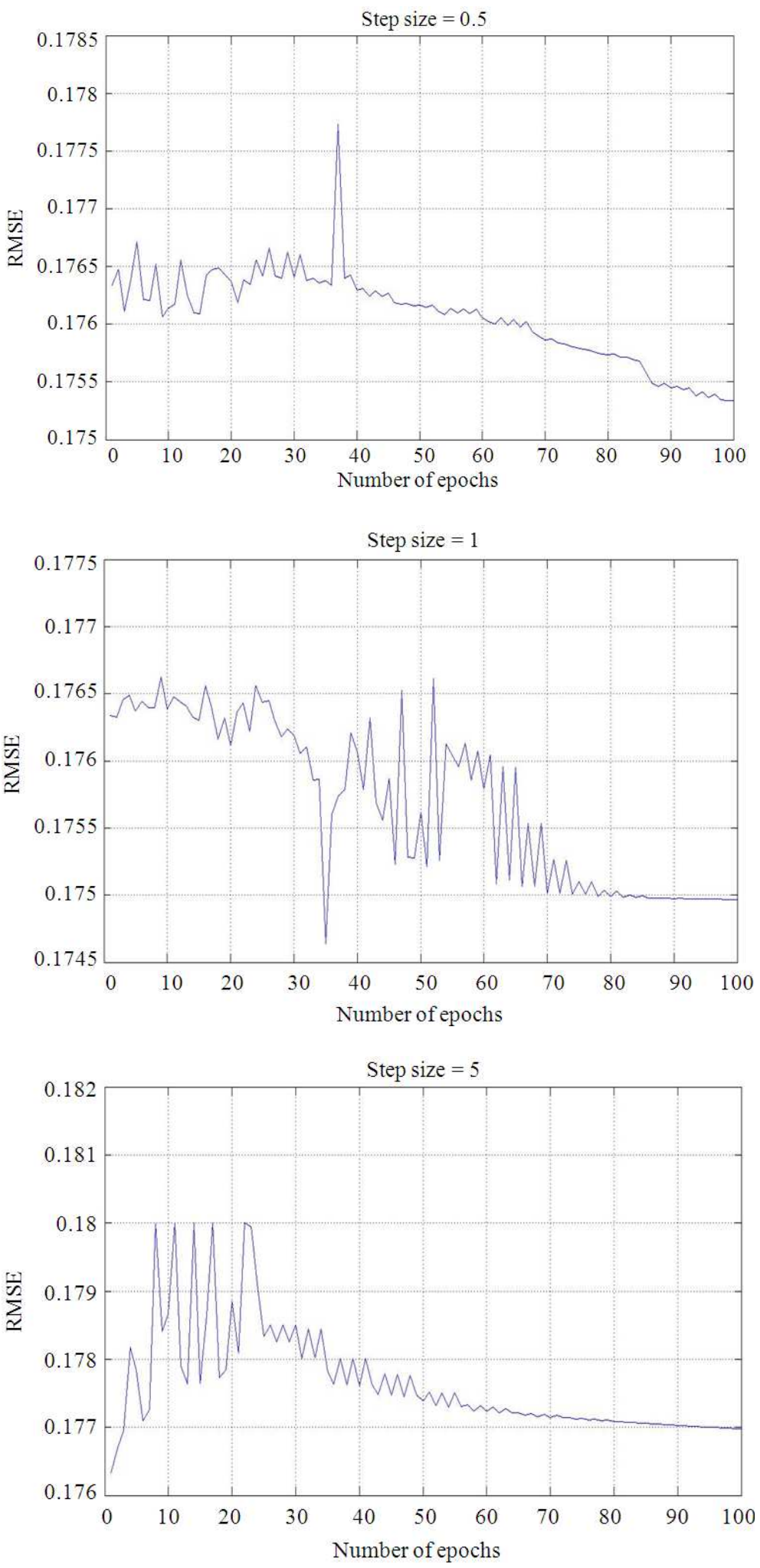

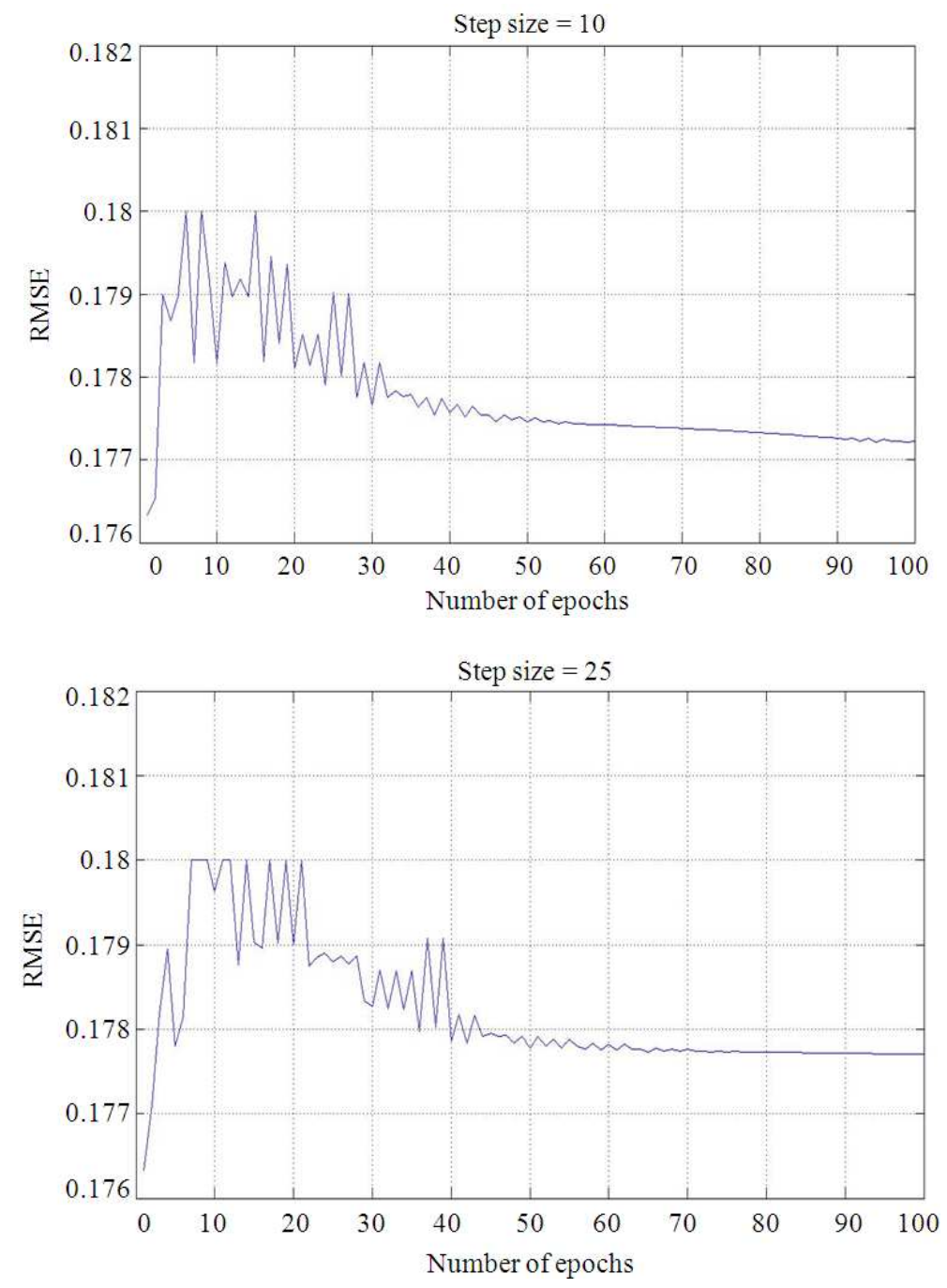

Fig. 5. Block diagram of ANFIS implementation in the GPS-aided INS

Results shown in Fig. 5 indicate that the selection of initial step size will greatly influence the achievable RMSE of the ANFIS training. It is also shown in Fig. 5 that the initial step size of 0.01 acquires the best training RMSE among the eight initial step sizes.

\section{RESULTS AND DISCUSSION}

This section outlines the implementation of the proposed ANFIS in the GPS-aided INS for short term GPS outage condition. The motion data from the UAV experiment, as shown in Fig. 6 using Google Earth, is being utilized for the no GPS condition implementation. The experiment was conducted in Kampung Seri Pantai,
Mersing, Malaysia. The duration of the experiments was approximately $50 \mathrm{~min}$. The average UAV velocity is 130 $\mathrm{km} / \mathrm{h}$ and the average number of satellites availability is 10 for the experiment.

Fig. 7 illustrates the ideal UAV's navigation path computed from the GPS-aided INS. Short durations of GPS outage are imposed in eight locations, as indicated by numerical numbers from 1 to 8 , as shown in Fig. 7 . These eight locations are carefully chosen to cover different UAV dynamics. The estimated position errors at all eight locations using the GPS-aided INS with and without ANFIS for short term GPS outage of $15 \mathrm{~s}, 20 \mathrm{~s}$, $25 \mathrm{~s}$ and $30 \mathrm{~s}$ are shown in Table 1. It could be observed from Table 1 that the estimated errors from the GPS- 
aided INS with ANFIS achieved better position estimation than the GPS-aided INS without ANFIS during short term no GPS conditions.

Fig. 8 illustrates the estimated position plots of the GPS-aided INS, with and without ANFIS, for short term GPS outage of $30 \mathrm{sec}$. The errors were computed with reference to the navigation path generated from the GPSaided INS without GPS outage. The errors were shown in Table 1, under the "30 s GPS Outage" row. It could be observed from Table 1 that the estimated errors from the GPS-aided INS with ANFIS implementation reduced significantly as compared to the GPS-aided INS without ANFIS at all the selected eight locations. Such result indicates that the GPS-aided INS with ANFIS implementation achieved better performance during GPS outage conditions.

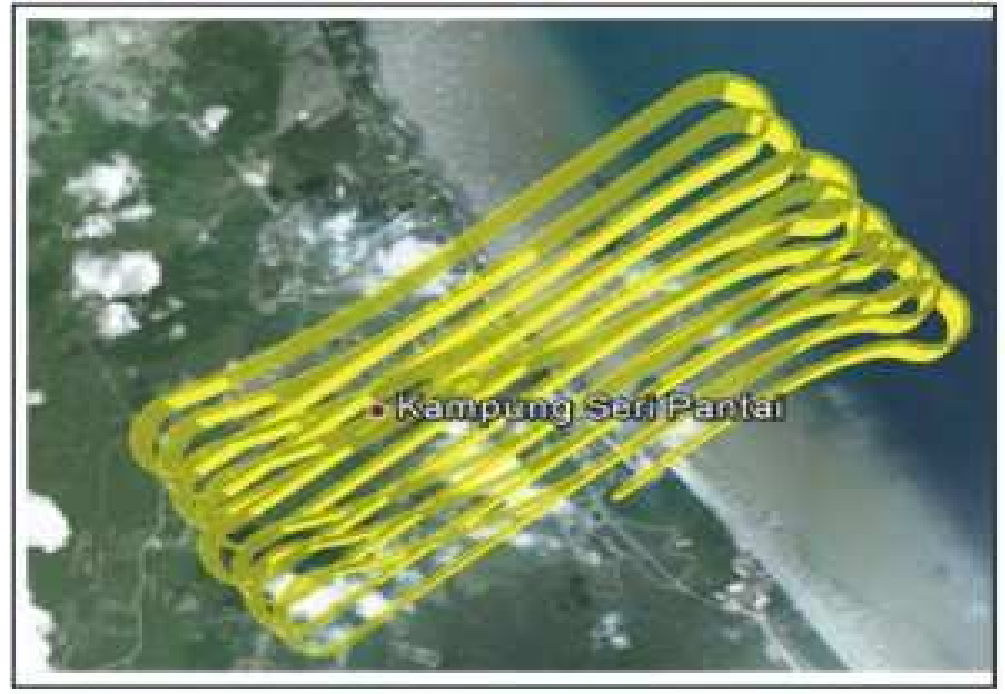

Fig. 6. UAV's navigation path using google earth

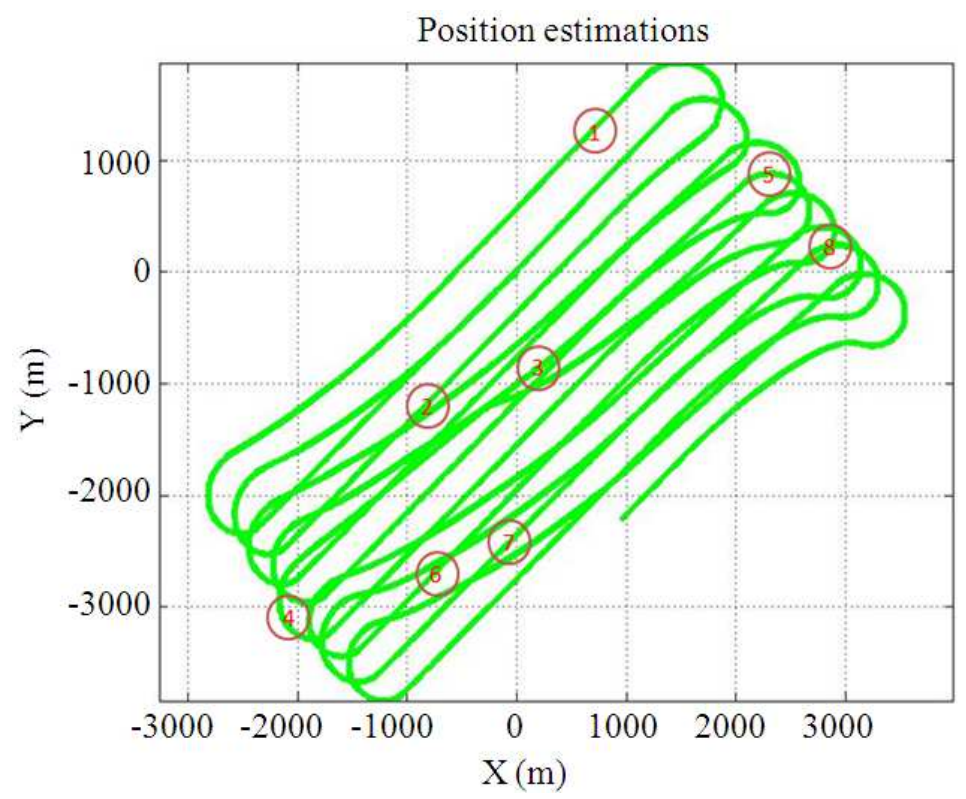

Fig. 7. UAV's navigation path with locations of intentionally introduced GPS outages 


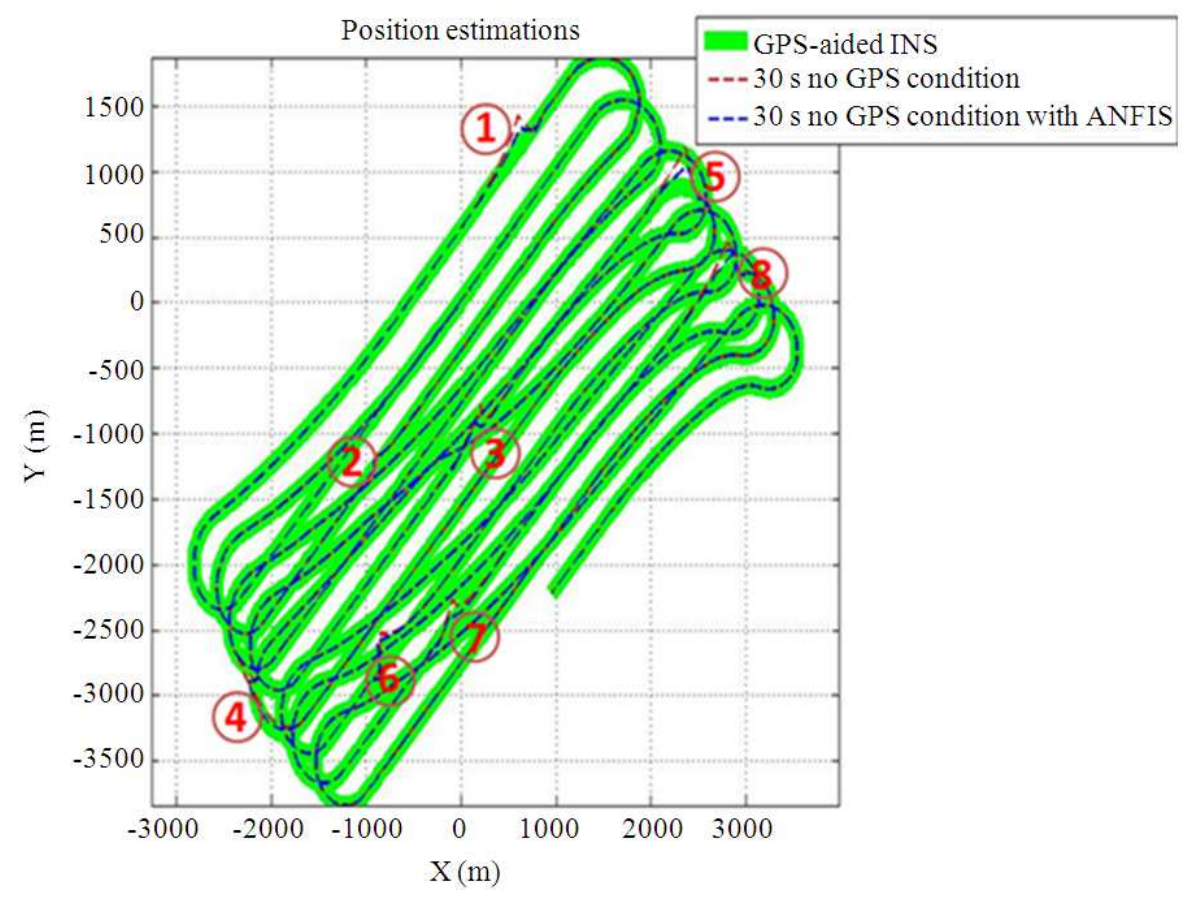

Fig. 8. Locations with 30 seconds of intentionally introduced GPS outages

Table 1. Estimated position errors with different GPS outage duration at different location

\begin{tabular}{|c|c|c|c|c|c|c|c|c|c|}
\hline \multirow[b]{2}{*}{ Locations } & & \multicolumn{8}{|c|}{ Position errors estimation (m) } \\
\hline & & 1 & 2 & 3 & 4 & 5 & 6 & 7 & 8 \\
\hline \multirow[t]{3}{*}{15 s GPS outage } & No ANFIS & 30.712 & 8.788 & 40.266 & 15.517 & 48.605 & 40.367 & 24.884 & 20.766 \\
\hline & With ANFIS & 26.223 & 6.944 & 27.066 & 11.492 & 32.829 & 39.294 & 22.302 & 9.847 \\
\hline & $\%$ Diff & $14.62 \%$ & $20.98 \%$ & $32.78 \%$ & $25.94 \%$ & $32.46 \%$ & $2.66 \%$ & $10.38 \%$ & $52.58 \%$ \\
\hline \multirow[t]{3}{*}{20 s GPS outage } & No ANFIS & 58.697 & 20.884 & 111.575 & 51.02 & 66.144 & 85.856 & 63.619 & 49.136 \\
\hline & With ANFIS & 46.53 & 15.426 & 79.796 & 29.13 & 38.769 & 76.073 & 33.336 & 28.589 \\
\hline & $\%$ Diff & $20.73 \%$ & $26.13 \%$ & $27.5 \%$ & $42.9 \%$ & $41.39 \%$ & $11.39 \%$ & $47.6 \%$ & 41.82 \\
\hline \multirow[t]{3}{*}{25 s GPS outage } & No ANFIS & 106.514 & 56.011 & 240.029 & 150.953 & 117.466 & 156.602 & 145.097 & 112.496 \\
\hline & With ANFIS & 77.574 & 36.139 & 174.294 & 82.631 & 38.842 & 131.1 & 76.492 & 66.519 \\
\hline & $\%$ Diff & $27.17 \%$ & $35.48 \%$ & $27.39 \%$ & $45.26 \%$ & $66.93 \%$ & $16.28 \%$ & $47.28 \%$ & $40.87 \%$ \\
\hline \multirow[t]{3}{*}{30 s GPS outage } & No ANFIS & 182.077 & 127.714 & 464.564 & 351.076 & 258.194 & 269.027 & 296.766 & 252.611 \\
\hline & With ANFIS & 122.04 & 77.839 & 331.151 & 211.797 & 79.901 & 213.248 & 171.383 & 145.064 \\
\hline & $\%$ Diff & $32.97 \%$ & $39.05 \%$ & $28.72 \%$ & $39.67 \%$ & $69.05 \%$ & $20.73 \%$ & $42.25 \%$ & $42.57 \%$ \\
\hline
\end{tabular}

\section{CONCLUSION}

This study presented the fundamental study of GPSaided INS and its limitation during short term GPS outage conditions. The ANFIS was proposed as a subremedy system to temporary replaces the GPS for motion sensing. Result signified that the GPS-aided INS with ANFIS implementation achieved better performance than the GPS-aided INS without ANFIS during short term GPS outage. For future work, the developed system will be implemented on an UAV Synthetic Aperture Radar (SAR) (Koo et al., 2012) for environmental monitoring applications. It is expected that the developed system is able to assist in the motion compensation of SAR image processing.

\section{ACKNOWLEDGEMENT}

This study is supported by Multimedia University and Telekom Malaysia under Research Fund RDTC/130824. 


\subsection{Author's Contributions}

All authors equally contributed in this work.

\subsection{Ethics}

This article is original and contains unpublished material. The corresponding author confirms that all of the other authors have read and approved the manuscript and no ethical issues involved.

\section{REFERENCES}

Adrian, H., L. René and F. Grancois, 2004. Fuzzy corrections in a GPS/INS hybrid navigation system. IEEE Trans. Aerospace Elect. Syst., 40: 591-600. DOI: 10.1109/TAES.2004.1310007

Ahmed, E.S., H. Aini and N.E. Aboelmagd, 2009. ANFIS-based model for real-time INS/GPS data fusion for vehicular navigation system. Proceedings of the International Conference on Computer Technology and Development, Nov. 13-15, IEEE Xplore Press, Kota Kinabalu, pp: 278-282. DOI: 10.1109/ICCTD.2009.42

Alison, K.B., 2005. GPS/INS uses low-cost MEMS IMU. IEEE Aerospace Elect. Syst. Mag., 20: 3-10. DOI: 10.1109/MAES.2005.1514768

Amari, S.I., N. Murata, K.R. Muller, M. Finke and H.H. Yang et al., 1997. Asymptotic statistical theory of overtraining and cross-validation. IEEE Trans. Neural Netw., 8: 985-996. DOI: 10.1109/72.623200

David, M.B., R. Jihan and J.C. Gerdes, 2006. Integrating INS sensors with GPS measurements for continuous estimation of vehicle sideslip, roll and tire cornering stiffness. IEEE Trans. Intelli. Transportat. Syst., 7: 483-493. DOI:10.1109/TITS.2006.883110

El-Diasty, M. and P. Spiros, 2008. Calibration and stochastic modelling of inertial navigation sensor errors. J. Global Position. Syst., 7: 170-182. DOI: 10.5081/jgps.7.2.170

Jang, J., 1993. ANFIS: Adaptive-network-based fuzzy inference system. IEEE Trans. Syst. Man Cybernet., 23: 665-685. DOI: 10.1109/21.256541

Koo, V.C., Y.K. Chan, V. Gobi, M.Y. Chua and B.C. Sew et al., 2012. A new unmanned aerial vehicle synthetic aperture radar for environmental monitoring. Progress Electromagnet. Res., 122: 245-268. DOI: 10.2528/PIER11092604
Lim, C.H., W.Q. Tan, T.S. Lim and V.C. Koo, 2012a. Practical approach in estimating inertial navigation unit's errors. IEICE Elect. Exp., 9: 772-778. DOI: 10.1587/elex.9.772

Lim, C.H., T.S. Lim and V.C. Koo, 2012b. Design and development of a real-time GPS-aided SINU system. Int. J. Adv. Robotic Syst., 9: 194-202. DOI: $10.5772 / 52681$

Lim, C.H., T.S. Lim and V.C. Koo. 2014. Stochastic error modeling of MEMS inertial sensor with implementation to GPS-aided INU system for UAV motion sensing. Applied Mechan. Mater., 464: 240-246. DOI: 10.4028/www.scientific.net/AMM.464.240

Naser, E.S., N. Sameh and N. Aboelmagd, 2004. Wavelet de-noising for IMU alighment. IEEE Aerospace Elect. Syst. Mag., 19: 32-39. DOI: 10.1109/MAES.2004.1365016

Nebot, E. and W.H. Durrant, 1999. Initial calibration and alignment of low cost inertial navigation units for land vehicle applications. J. Robotics Syst., 16: 81-92. DOI: $10.1002 /($ SICI)10974563(199902)16:2<81::AID-ROB2>3.0.CO;2-9

Rashad, S., R.T. Mahmoud, T. Mohammed and N. Aboelmagd, 2007. Merits and limitations of using fuzzy inference system for temporal integration of INS/GPS in vehicular navigation. Soft Comput., 11: 889-900. DOI: 10.1007/s00500-006-0140-0

Sameh, N., 2003. Improving the Inertial Navigation System (INS) error model for INS and INS/DGPS applications. Unpublished dissertation in partial fulfillment of the requirements for the degree of Doctor of Philosophy, Department of Geomatics Engineering. The University of Calgary, Alberta, Canada.

Sheimy, N.E., H. Hou and X. Niu, 2008. Analysis and modeling of inertial sensors using allan variance. IEEE Trans. Instrumentat. Measurement, 57: 140149. DOI: 10.1109/TIM.2007.908635

Titterton, D.H. and J.L. Weston, 1997. Strapdown Inertial Navigation Technology. 2nd Edn., Institution of Electrical Engineers, Stevenage, ISBN-10: 0863413587, pp: 558. 